\title{
Increased risk of infection with SARS-CoV-2 Omicron BA.1 compared with Delta in vaccinated and previously infected individuals, the Netherlands, 22 November 2021 to 19 January 2022
}

\author{
${\text { Dirk Eggink }{ }^{1, *}, \text { Stijn P Andeweg }}^{1, *}$, Harry Vennema ${ }^{1}$, Noortje van Maarseveen ${ }^{2,3}$, Klaas Vermaas $^{2}$, Boris Vlaemynck ${ }^{4}$, Raf \\ Schepers $^{4}$, Arianne B van Gageldonk-Lafeber ${ }^{1}$, Susan van den Hof ${ }^{1}$, Chantal BEM Reusken ${ }^{1, * *}$, Mirjam J Knol ${ }^{1, * \star}$ \\ 1. Center for Infectious Disease Control, WHO COVID-19 reference laboratory, National Institute for Public Health and the \\ Environment (RIVM), Bilthoven, The Netherlands \\ 2. Saltro Diagnostic Center for Primary Care, Utrecht, The Netherlands \\ 3. Department of Medical Microbiology, University Medical Center Utrecht, Utrecht, the Netherlands \\ 4. SYNLAB, Heppignies, Belgium \\ * These authors contributed equally to this article and share first authorship. \\ ** These authors contributed equally to this article and share last authorship.
}

Correspondence: Dirk Eggink (dirk.eggink@rivm.nl)

Citation style for this article:

Eggink Dirk, Andeweg Stijn P, Vennema Harry, van Maarseveen Noortje, Vermaas Klaas, Vlaemynck Boris, Schepers Raf, van Gageldonk-Lafeber Arianne B, van den Hof Susan, Reusken Chantal BEM, Knol Mirjam J. Increased risk of infection with SARS-CoV-2 Omicron BA.1 compared with Delta in vaccinated and previously infected individuals, the Netherlands, 22 November 2021 to 19 January 2022. Euro Surveill. 2022;27(4):pii=2101196. https://doi.org/10.2807/1560-7917.

ES.2022.27.4.2101196

Infections with the Omicron SARS-CoV-2 variant are rapidly increasing worldwide. Among 174,349 SARSCoV-2-infected individuals ( $\geq 12$ years), we observed an increased risk of $\mathrm{S}$ gene target failure, predictive of the Omicron variant, in vaccinated (odds ratio (OR): 3.6; $95 \%$ confidence interval $(\mathrm{CI}): 3.4-3.7)$ and previously infected individuals (OR: $4.2 ; 95 \% \mathrm{Cl}: 3.8-4.7$ ) compared with infected naïve individuals. This suggests vaccine- or infection-induced immunity against SARS-CoV-2 infections is less effective against the Omicron than the Delta variant.

On 26 November 2021, the World Health Organization (WHO) declared the Omicron variant (Phylogenetic Assignment of Named Global Outbreak Lineages (Pangolin) designation B.1.1.529) of severe acute respiratory syndrome coronavirus 2 (SARS-CoV-2) a variant of concern [1]. Globally, infections caused by Omicron have increased rapidly, showing higher transmissibility of this variant $[2,3]$. Omicron became the dominant variant in parts of Europe in early 2022 [4]. It displays a number of alterations in the spike protein, including around 30 amino acid substitutions, three deletions and one insertion [5-7]. These alterations raise concerns about the protection evoked by current SARS-CoV-2 vaccines against the Omicron variant.

To explore possible escape from vaccine- or infectioninduced immunity by Omicron compared with Delta, we here investigate the distribution of the Delta (B.1.617.2) and Omicron variants among SARS-CoV-2-positive individuals who had been naïve, had received the complete primary vaccination series or had a previous infection. We employed a case-only approach in which we compared the immune status among cases infected with the Omicron vs the Delta variant. We thereby assess the relative effectiveness of vaccination against Omicron vs Delta viruses [8]. Similarly, we analyse if the protective effect of previous SARS-CoV-2 infection is different against a new infection with Delta vs Omicron viruses.

\section{$S$ gene target failure as a measure for Omicron infection}

We used data from two large diagnostic laboratories analysing specimens from national community testing in the Netherlands that make use of the TaqPath COVID19 RT-PCR kit (ThermoFisher Scientific, Nieuwegein, The Netherlands). The Omicron (BA.1) but not the Delta variant, with the exception of some very sporadically circulating Delta variant strains, possesses a deletion at amino acid positions 69 and 70 of the spike protein $(\Delta 69-70)$ that has been associated with failure of the probe targeting the $\mathrm{S}$ gene, while the ORF1ab and $\mathrm{N}$ probes result in a positive signal (S gene target failure (SGTF), also referred to as $\mathrm{S}$ drop-out). This failure of detection of the $\mathrm{S}$ gene target in an otherwise positive PCR test has proven to be a highly specific proxy for the presence of the Alpha variant (B.1.1.7) in the past and also identifies Omicron [9-12]. However, with lower viral loads, the $\mathrm{S}$ gene target tends to be the least 
Number of included COVID-19 cases and percentage of S gene target failures by immune status, the Netherlands, 22 November 2021-19 January $2022(\mathrm{n}=174,349)$

\section{A. Included Covid-19 cases}

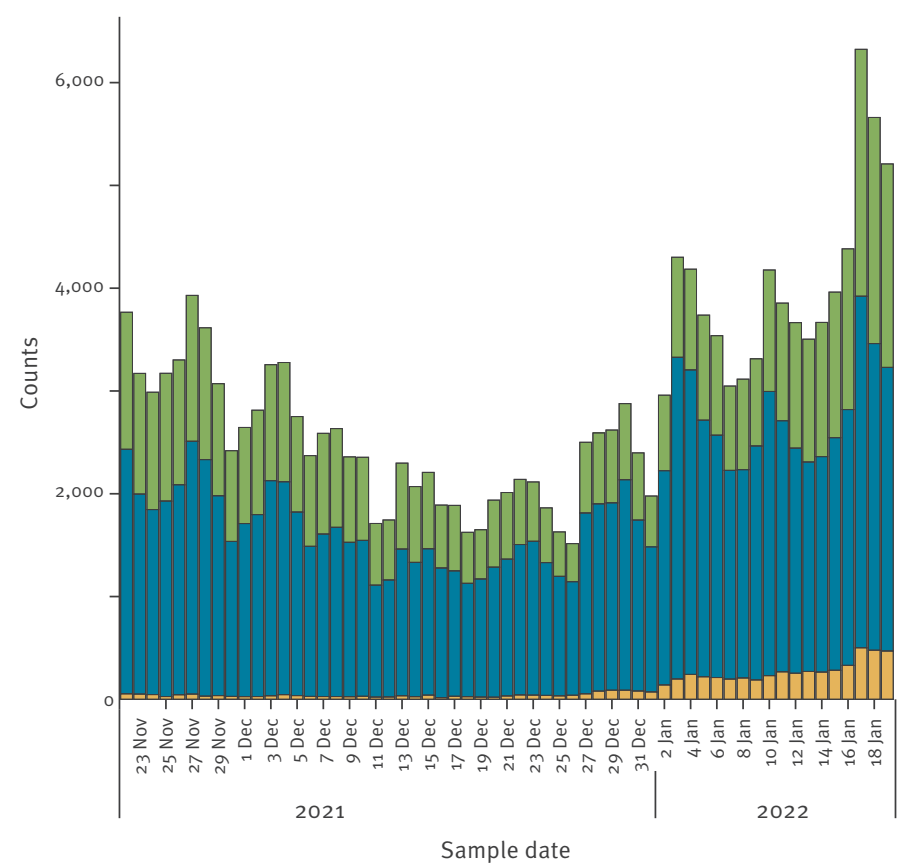

B. S gene target failures

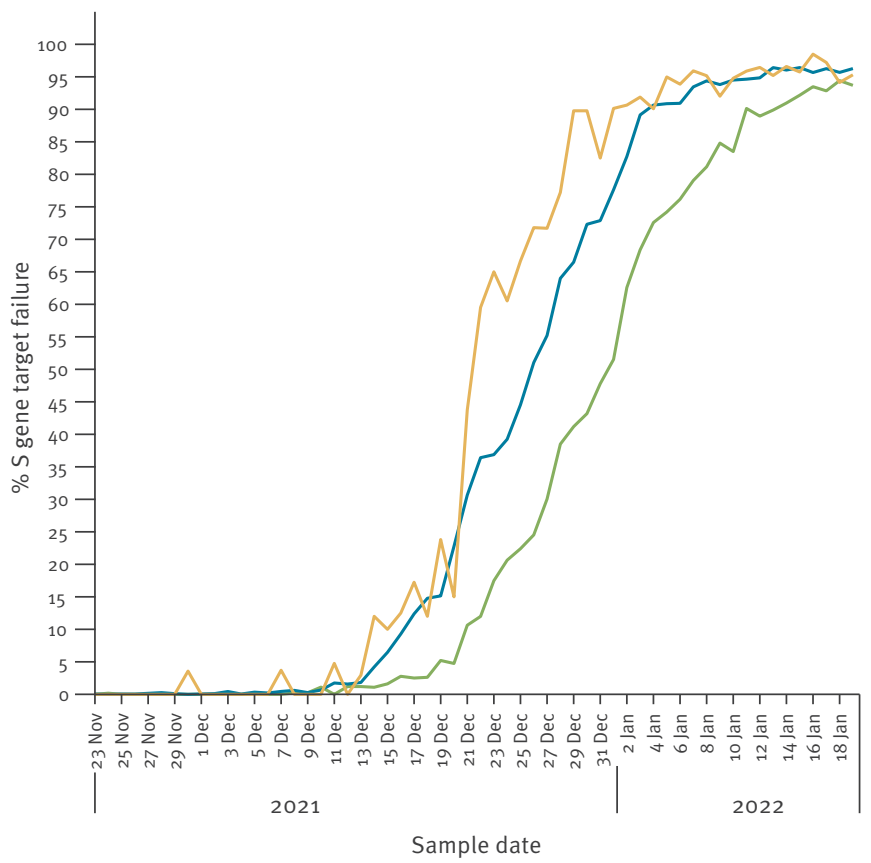

Immune status $\square$ Naive $\square$ Vaccinated $\square$ Previous infection

COVID-19: coronavirus disease.

sensitive of the three targets in the Taqpath system, which could result in incorrect interpretation of an SGTF result. To avoid false-positive SGTF results, we used a stringent threshold to identify likely Omicron infections. In-house titration showed decreased sensitivity for the $S$ gene target in case of viral loads that gave a quantification cycle $(\mathrm{Cq})$ value of around 32 or more for the ORF1ab and $\mathrm{N}$ targets. Therefore, only results with a Cq value $\leq 30$ for both targets were included for further analyses.

Sequencing of a random selection of SGTF samples included in this study confirmed the presence of the Omicron variant (BA.1) in 325 of 329 cases (98.8\%). Alpha $(n=1)$ and Delta $(n=3)$ variants containing the $\Delta 69-70$ were detected in the remaining samples. The correlation between SGTF and Omicron increased over time, with only Omicron detections in the SGTF samples after 5 December 2021.

S gene testing results were linked to vaccination status data from the national notification register (OSIRIS), which includes all SARS-CoV-2 cases in the Netherlands. If vaccination status was unknown from the notification register, we used self-reported vaccination status from the community testing register (CoronIT). A person was defined as having completed the primary vaccination series when they had received two doses of Comirnaty (BNT162b2, BioNTech/Pfizer, Mainz, Germany/New York, United States (US)), Spikevax (mRNA-1273, Moderna, Cambridge, US) or Vaxzevria (ChAdOx1, AstraZeneca, Cambridge, United Kingdom) more than 14 days before the symptom onset date or one dose of COVID-19 Vaccine Janssen (JanssenCilag International NV, Beerse, Belgium) more than 28 days before the symptom onset date. For asymptomatic cases, we used sample date $(6,165$ of 110,965 vaccinated cases) and for symptomatic cases or unknown symptoms status for whom onset date was missing, we used the sample date minus 2 days $(19,136$ of 110,965 vaccinated cases). People who had not received any vaccine were defined as unvaccinated. Previous infection was defined as a positive PCR or antigen test result at least 8 weeks before the current positive test, based on the national notification and testing register. We excluded childrenyounger than 12 years because they had not been eligible for vaccination in the study period and there had been different testing policies in this age group over time, influencing the likelihood of detecting a previous infection.

\section{Statistical analysis}

Among infected individuals, we compared the percentage of SGTF results between unvaccinated cases without a known previous infection (naïve), primary vaccinated cases without a known previous infection, 
Characteristics of SARS-CoV-2 cases 12 years and older with S gene detected and S gene target failure, the Netherlands, 22 November 2021-19 January $2022(\mathrm{n}=174,349)$

\begin{tabular}{|c|c|c|c|c|}
\hline \multirow{3}{*}{ Total } & \multicolumn{2}{|c|}{ S gene detected } & \multicolumn{2}{|c|}{ S gene target failure } \\
\hline & $\mathrm{n}$ & $\%$ & $\mathrm{n}$ & $\%$ \\
\hline & 93,734 & 100 & 80,615 & 100 \\
\hline \multicolumn{5}{|l|}{ Immune status } \\
\hline Naïve & 34,765 & 37.1 & 22,071 & 27.4 \\
\hline Primary vaccination & 57,674 & 61.5 & 53,291 & 66.1 \\
\hline Previous infection & 1,295 & 1.4 & 5,253 & 6.5 \\
\hline \multicolumn{5}{|l|}{ Age group (years) } \\
\hline $12-19$ & 11,338 & 12.1 & 21,231 & 26.3 \\
\hline $20-29$ & 13,907 & 14.8 & 22,676 & 28.1 \\
\hline $30-39$ & 18,028 & 19.2 & 13,554 & 16.8 \\
\hline $40-49$ & 16,871 & 18.0 & 9,815 & 12.2 \\
\hline $50-59$ & 13,872 & 14.8 & 9,223 & 11.4 \\
\hline $60-69$ & 11,427 & 12.2 & 3,009 & 3.7 \\
\hline $70-79$ & 6,501 & 6.9 & 941 & 1.2 \\
\hline$\geq 80$ & 1,790 & 1.9 & 166 & 0.2 \\
\hline \multicolumn{5}{|l|}{ Sex } \\
\hline Male & 47,172 & 50.3 & 39,981 & 49.6 \\
\hline Female & 46,562 & 49.7 & 40,634 & 50.4 \\
\hline \multicolumn{5}{|l|}{ Travel history } \\
\hline Yes & 1,000 & 1.1 & 4,162 & 5.2 \\
\hline No & 43,630 & 46.5 & 21,363 & 26.5 \\
\hline Unknown & 49,104 & 52.4 & 55,090 & 68.3 \\
\hline Interval & Median & IQR & Median & IQR \\
\hline Number of days between onset and last vaccination & 155 & $130-183$ & 157 & $139-180$ \\
\hline Number of days between onset and previous infection & 343 & $246-396$ & 274 & $170-390$ \\
\hline
\end{tabular}

IQR: interquartile range; SARS-CoV-2: severe acute respiratory syndrome coronavirus 2.

and unvaccinated cases with a known previous infection. The number of primary vaccinated persons with a previous infection was small and therefore excluded. We performed logistic regression to estimate the association between immune status and SGTF, adjusting for testing date, 10-year age group and sex. In addition, we stratified the analyses by three age groups (12-29, 30-59 and $\geq 60$ years) and adjusted for testing date, 5 -year age group and sex. If the Omicron and Delta variants had similar ability to escape immunity from vaccination or previous infection, we would expect the same proportion of SGTF in vaccinated or previously infected persons as in naïve individuals, i.e. an odds ratio (OR) of 1 . As travel history could be related to being infected with the Omicron variant and with being vaccinated, we performed an additional analysis where we excluded cases with a history of travel outside the Netherlands in the 14 days before symptom onset.

\section{Immune status and $S$ gene target failure}

Between 22 November 2021 and 19 January 2022, 174,349 PCR-positive samples with a Cq value $\leq 30$ for ORF1ab and $\mathrm{N}$ gene targets were analysed for SGTF and included in the current analysis. SGTF was present in
$80,615$ cases ( $46.2 \%)$; the percentage of SGTF increased rapidly from early December (Figure).

Cases with SGTF were younger and more often had a travel history (Table 1). Of the SGTF cases, 53,291 $(66.1 \%)$ had received primary vaccination compared with $61.5 \%(n=57,674)$ of non-SGTF cases, and $6.5 \%$ $(n=5,253)$ had a previous infection compared with $1.4 \%(1,295)$ of the non-SGTF cases. Correspondingly, we found an adjusted OR (AOR) of 3.6 (95\% confidence interval $(\mathrm{Cl}): 3.4-3.7)$ for the association between full vaccination and SGTF (Table 2). The AOR for previous infection was $4.2(95 \% \mathrm{Cl}: 3.8-4.7)$. When excluding cases with any known travel history, the OR remained the same for previous infection $(\mathrm{OR}=4.2$; $95 \% \mathrm{Cl}: 3.8-$ 4.7) and was slightly lower but still significant for vaccination $(\mathrm{OR}=3.4 ; 95 \% \mathrm{Cl}: 3.2-3 \cdot 5)$.

Stratified by age group, the AOR for primary vaccination were 4.1 (95\% Cl: 3.9-4.4), 3.2 (95\% Cl: 3.0-3.4) and 2.8 (95\% Cl: $2.3-3.2)$ for $12-29$ years, 30-59 years and $\geq 60$ years, respectively. For previous infection, the AOR were 3.7 (95\% Cl: 3.2-4.3), 4.8 (95\% Cl: 4.0-5.7) and 6.6 (95\% Cl: 3.5-13.0) for $12-29$ years, 30-59 years and $\geq 60$ years, respectively. 


\section{TABLE 2}

Association between immune status and S gene target failure, adjusted for day of sampling, sex and 10-year age group, the Netherlands, 22 November 2021-19 January $2022(\mathrm{n}=174,349)$

\begin{tabular}{|l|c|c|}
\hline \multirow{2}{*}{ Immune status } & All data & Without travel history \\
\cline { 2 - 3 } & OR $(95 \% \mathrm{Cl})$ & OR $(95 \% \mathrm{Cl})$ \\
\hline Naïve & Reference & Reference \\
\hline Primary vaccination & $3.6(3.4-3.7)$ & $3.4(3.2-3.5)$ \\
\hline Previous infection & $4.2(3.8-4.7)$ & $4.2(3.8-4.7)$ \\
\hline
\end{tabular}

$\mathrm{Cl}$ : confidence interval; OR: odds ratio.

\section{Ethical statement}

The Centre for Clinical Expertise at the National Institute for Public Health and the Environment (RIVM) assessed the research proposal following the specific conditions as stated in the law for medical research involving human subjects. The work described was exempted for further approval by the ethical research committee. Pathogen surveillance is a legal task of the RIVM and is carried out under the responsibility of the Dutch Minister of Health, Welfare and Sports. The Public Health Act (Wet Publieke Gezondheid) provides that RIVM may receive pseudonymised data for this task without informed consent.

\section{Discussion}

Our results suggest a large reduction in protection against SARS-CoV-2 infection with the Omicron variant, as measured by SGTF, compared with the Delta variant after primary vaccination. This is in accordance with in vitro studies that showed a 30-40-fold reduction in neutralisation of the Omicron variant compared with wild type when using convalescent sera or sera of individuals who had completed the primary vaccination series [5-7]. Several studies have reported a substantial reduction in vaccine effectiveness against infection with the Omicron variant compared with Delta after primary vaccination [13-16]. After booster vaccination, the vaccine effectiveness against infection with the Omicron variant increased but was still lower than against Delta [14-18]. Studies have shown that vaccine effectiveness against severe COVID-19 with the Omicron variant is very high after booster vaccination, although lower than against severe COVID-19 with Delta $[19,20]$.

We showed that individuals with a previous infection had a higher risk of infection with Omicron than with Delta compared with naïve individuals, suggesting that previous infection with another SARS-CoV-2 variant provides lower levels of protection against Omicron than against Delta infection. This contrasts with what we observed in a previous analysis, where we did not find an increased risk of infection with the Delta, Beta (B.1.351) and Gamma (P.1) variants vs the Alpha variant in previously infected individuals [21]. Our finding of reduced protection against reinfection with Omicron is in line with reports showing increased risk of reinfections with the Omicron variant [22-24].

We found a lower OR for vaccination in older age groups, suggesting that the difference in vaccine effectiveness against Omicron versus Delta is lower with higher age. We do not have an explanation for this observation and therefore this may need further investigation.

Our study looked at infections based on community testing. Several studies suggest that infection with the Omicron variant causes less severe disease, with lower rates of hospitalisations and intensive care admission than for Delta infection, also when considering the effect of vaccination and previous infection $[12,25,26]$. The effectiveness of primary vaccination against hospitalisation with Omicron infection was reported to be around $50 \%$ at 25 weeks or more after vaccination, and the effectiveness increased to ca 90\% within 2-9 weeks after booster vaccination $[19,20]$. Even with lower disease severity for Omicron compared with Delta, the higher transmissibility will probably lead to more hospital admissions if no additional measures are taken.

A strength of a case-only design is that it prevents bias from poor control selection and it prevents bias from exposure misclassification differential by disease status, as it only includes people with the disease. The method we used is recommended by the WHO to assess the impact of new variants on vaccine effectiveness [8]. Our study also has some limitations. We relied on reported previous infections, and therefore some misclassification will have occurred as not all previous infections are detected and hence reported. Also using self-reported vaccination status may have led to some misclassification, although it is unlikely that this would be different in people infected with the Omicron compared with the Delta variant. We used SGTF as a proxy for Omicron. Omicron was confirmed by sequencing in $99 \%$ of 329 samples, so limited misclassification due to use of SGTF can be expected to be limited. Of note, SGTF can discriminate between Delta and the BA.1 but not the BA.2 variant of Omicron. At the end of our study period, BA.2 had only been rarely detected in the Netherlands. For our study design to be valid, we have to assume that vaccination and previous infection are independent of variant status, i.e. there should not be a third factor influencing both vaccination and having an Omicron infection. This is questionable for people with a travel history, as vaccination might be a requirement to allow travel. A sensitivity analysis excluding cases with any travel history did not substantially change our results. We have not yet been able to assess the effect of booster vaccination which has only recently started in the Netherlands (18 November 2021). 


\section{Conclusion}

Our results suggest a large decrease in protection from vaccine- or infection-induced immunity against SARS-CoV-2 infections caused by the Omicron variant compared with the Delta variant. This emphasises the need of booster vaccination and will warrant implementing non-pharmaceutical interventions to prevent overwhelming hospital care if COVID-19 severity is not reduced to a great extent.

\section{Conflict of interest}

None declared.

\section{Authors' contributions}

DE, SPA, CBEMR, MJK designed the study; HV, NvM, KV, BV, RS generated the data; DE, SPA, MJK performed analyses; ABvGL, SvdH, CBEMR,MJK supervised the study; DE, SA, $A B v G L, C B E M R, M J K$ and wrote the manscript.

\section{References}

1. World Health Organization (WHO). Tracking SARSCoV-2 variants. Geneva: WHO. [Accessed: 30 Dec 2021]. Available from: https://www.who.int/en/activities/ tracking-SARS-CoV-2-variants

2. European Centre for Disease Prevention and Control (ECDC). Epidemiological update: Omicron variant of concern (VOC) data as of 16 December 2021 (12:00). Stockholm: ECDC; 2021. Available from: https://www.ecdc.europa.eu/en/news-events/ epidemiological-update-omicron-data-16-december

3. Barnard RC, Davies N, Pearson CAB, Jit M, Edmunds J. Modelling the potential consequences of the Omicron SARSCoV-2 variant in England | Centre for Mathematical Modelling of Infectious Diseases Repository. London: London School of Hygiene \& Tropical Medicine; 2021. Preprint. Available from: https://cmmid.github.io/topics/covid19/omicron-england.html

4. European Centre for Disease Prevention and Control (ECDC). Weekly epidemiological update: Omicron variant of concern (VOC) - week 2 (data as of 20 January 2022) EU/EEA. Stockholm: ECDC; 2022. Available from: https://www.ecdc. europa.eu/en/news-events/weekly-epidemiological-updateomicron-variant-concern-voc-week-2-data-20-january-2022

5. Cele S, Jackson L, Khoury DS, Khan K, Moyo-Gwete T, Tegally $\mathrm{H}$, et al. Omicron extensively but incompletely escapes Pfizer BNT162b2 neutralization. Nature. 2021. https://doi. org/10.1038/s41586-021-04387-1 PMID: 35016196

6. Callaway E. Heavily mutated Omicron variant puts scientists on alert. Nature. 2021;600(7887):21-21. https://doi.org/10.1038/ d41586-021-03552-w PMID: 34824381

7. Wilhelm A, Widera M, Grikscheit K, Toptan T, Schenk B, Pallas C, et al. Reduced neutralization of SARS-CoV-2 Omicron variant by vaccine sera and monoclonal antibodies. medRxiv.2021.12.07.21267432. preprint. https://doi.org/ https://doi.org/10.1101/2021.12.07.21267432

8. World Health Organization (WHO). Guidance on conducting vaccine effectiveness evaluations in the setting of new SARSCoV-2 variants: Interim guidance, 22 July 2021. Addendum to Evaluation of COVID-19 vaccine effectiveness. Geneva: WHO: 2021. Available from: https://www.who.int/publications/i/ item/WHO-2019-nCoV-vaccine_effectiveness-variants-2021.1

9. Volz E, Mishra S, Chand M, Barrett JC, Johnson R, Geidelberg $\mathrm{L}$, et al. Assessing transmissibility of SARS-CoV-2 lineage B.1.1.7 in England. Nature. 2021;593(7858):266-9. https://doi. org/10.1038/s41586-021-03470-x PMID: 33767447

10. Borges V, Sousa C, Menezes L, Gonçalves AM, Picão M, Almeida JP, et al. Tracking SARS-CoV-2 lineage B.1.1.7 dissemination: insights from nationwide spike gene target failure (SGTF) and spike gene late detection (SGTL) data, Portugal, week 492020 to week 3 2021. Euro Surveill. 2021;26(10):2100131. https://doi.org/10.2807/1560-7917. ES.2021.26.10.2100130 PMID: 33706862

11. Washington NL, Gangavarapu K, Zeller M, Bolze A, Cirulli ET, Schiabor Barrett KM, et al. Emergence and rapid transmission of SARS-CoV-2 B.1.1.7 in the United States.
Cell. 2021;184(10):2587-2594.e7. https://doi.org/10.1016/j. cell.2021.03.052 PMID: 33861950

12. UK Health Security Agency. SARS-CoV-2 variants of concern and variants under investigation in England, Technical briefing 33. London: UK Health Security Agency; 2021. Available from: https://assets.publishing.service.gov.uk/government/uploads/ system/uploads/attachment_data/file/1043807/technicalbriefing-33.pdf

13. Garcia-Beltran WF, St Denis KJ, Hoelzemer A, Lam EC, Nitido $A D$, Sheehan ML, et al. mRNA-based COVID-19 vaccine boosters induce neutralizing immunity against SARS-CoV-2 Omicron variant. Cell. 2022;So092-8674(21)01496-3. https://doi. org/10.1016/j.cell.2021.12.033 PMID: 34995482

14. Andrews N, Stowe J, Kirsebom F, Toffa S, Rickeard T, Gallagher E, et al. Effectiveness of COVID-19 vaccines against the Omicron (B.1.1.529) variant of concern. medRxiv.2021.12.14.21267615. preprint. https://doi.org/10.110 1/2021.12.14.21267615

15. Hansen $\mathrm{CH}$, Schelde AB, Moustsen-Helm IR, Emborg H-D, Krause TG, Mølbak K, et al. Vaccine effectiveness against SARS-CoV-2 infection with the Omicron or Delta variants following a two-dose or booster BNT162 b2 or mRNA-1273 vaccination series: A Danish cohort study. medRxiv.2021.12.20.21267966. preprint. https://doi.org/10.1 101/2021.12.20.21267966 https://doi.org/10.1101/2021.12.20 .21267966

16. Tseng HF, Ackerson BK, Luo Y, Sy LS, Talarico CA, Tian Y, et al. Effectiveness of mRNA-1273 against SARS-CoV-2 omicron and delta variants. medRxiv.2022.01.07.22268919. preprint. https://doi.org/10.1101/2022.01.07.22268919 https://doi.org/ 10.1101/2022.01.07.22268919

17. Sheikh A, Kerr S, Woolhouse M, Mcmenamin J, Robertson C. Severity of Omicron variant of concern and vaccine effectiveness against symptomatic disease: national cohort with nested test negative design study in Scotland. Edinburgh: University of Edinburgh; 2021. Preprint. Available from: https://www.research.ed.ac.uk/en/publications/severity-ofomicron-variant-of-concern-and-vaccine-effectiveness-

18. UK Health Security Agency. SARS-CoV-2 variants of concern and variants under investigation - Technical briefing 34 . London: UK Health Security Agency; 2022. Available from: https://assets.publishing.service.gov.uk/government/uploads/ system/uploads/attachment_data/file/1048395/technicalbriefing-34-14-january-2022.pdf

19. Thompson MG, Natarajan K, Irving SA, Rowley EA, Griggs EP, Gaglani M, et al. Effectiveness of a third dose of mRNA vaccines against COVID-19-associated emergency department and urgent care encounters and hospitalizations among adults during periods of Delta and Omicron variant predominance VISION Network, 10 States, August 2021-January 2022. MMWR Morb Mortal Wkly Rep. 2022;71(4). https://doi.org/10.15585/ mmwr.mm7104e3

20. UK Health Security Agency. COVID-19 vaccine surveillance report - week 3. London: UK Health Security Agency; 2022. Available from: https://assets.publishing.service.gov.uk/ government/uploads/system/uploads/attachment_data/ file/104916o/Vaccine-surveillance-report-week-3-2022.pdf

21. Andeweg SP, Vennema H, Veldhuijzen I, Smorenburg $\mathrm{N}$, Schmitz D, Zwagemaker F, et al. Increased risk of infection with SARS-CoV-2 Beta, Gamma, and Delta variant compared to Alpha variant in vaccinated individuals. medRxiv.2021.11.24.21266735. preprint. https://doi.org/10.11 01/2021.11.24.21266735

22. Pulliam IRC, van Schalkwyk C, Govender N, von Gottberg A, Cohen C, Groome MJ, et al. Increased risk of SARS-CoV-2 reinfection associated with emergence of the Omicron variant in South Africa. medRxiv.2021.11.11.21266068. preprint. https://doi.org/10.1101/2021.11.11.21266068

23. Head E, van Elsland SL. Omicron largely evades immunity from past infection or two vaccine doses. London: Imperial College; 2021. Available from: https://www.imperial.ac.uk/news/232698/ modelling-suggests-rapid-spread-omicron-england

24. Altarawneh H, Chemaitelly H, Tang P, Hasan MR, Qassim $\mathrm{S}$, Ayoub HH, et al. Protection afforded by prior infection against SARS-CoV-2 reinfection with the Omicron variant. medRxiv.2022.01.05.22268782. preprint. https://doi.org/10.11 01/2022.01.05.22268782

25. Lewnard JA, Hong VX, Patel MM, Kahn R, Lipsitch M, Tartof SY. Clinical outcomes among patients infected with Omicron (B.1.1.529) SARS-CoV-2 variant in southern California. medRxiv.2022.01.11.22269045. preprint. https://doi.org/10.11 01/2022.01.11.22269045

26. Wolter N, Jassat W, Walaza S, Welch R, Moultrie H, Groome $M$, et al. Early assessment of the clinical severity of the SARS-CoV-2 omicron variant in South Africa: a data linkage 
study. Lancet. 2022;S0140-6736(22)00017-4. https://doi. org/10.1016/S0140-6736(22)00017-4

License, supplementary material and copyright

This is an open-access article distributed under the terms of the Creative Commons Attribution (CC BY 4.0) Licence. You may share and adapt the material, but must give appropriate credit to the source, provide a link to the licence and indicate if changes were made.

Any supplementary material referenced in the article can be found in the online version.

This article is copyright of the authors or their affiliated institutions, 2022. 